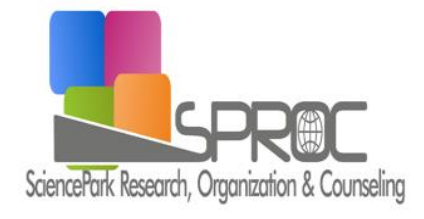

$$
\begin{array}{ccc}
\mathrm{k} & \mathrm{kH} & \mathrm{p} \\
\mathrm{ck} & \mathrm{h}
\end{array}
$$

H

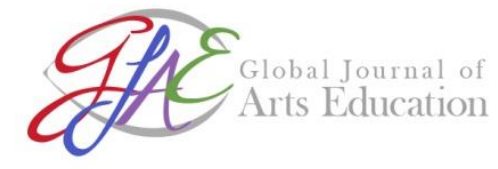

www.gjae.eu

\title{
Ceramics as the conveyance of art
}

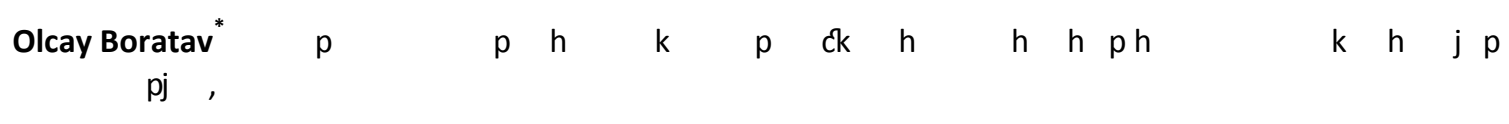

\section{Suggested Citation:}

$\mathrm{p}, \mathrm{p} \mathrm{h} \quad \mathrm{p}$, Global Journal of Arts Education. 6

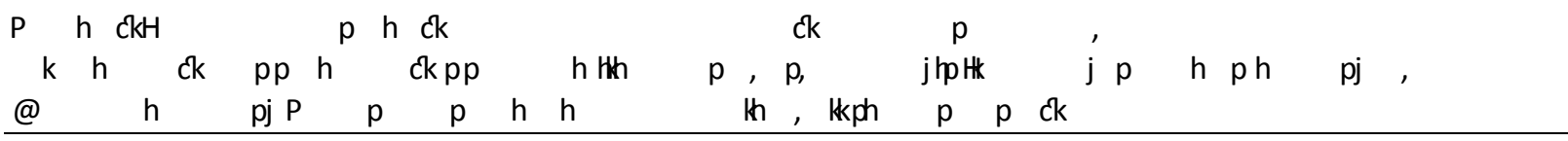

Abstract

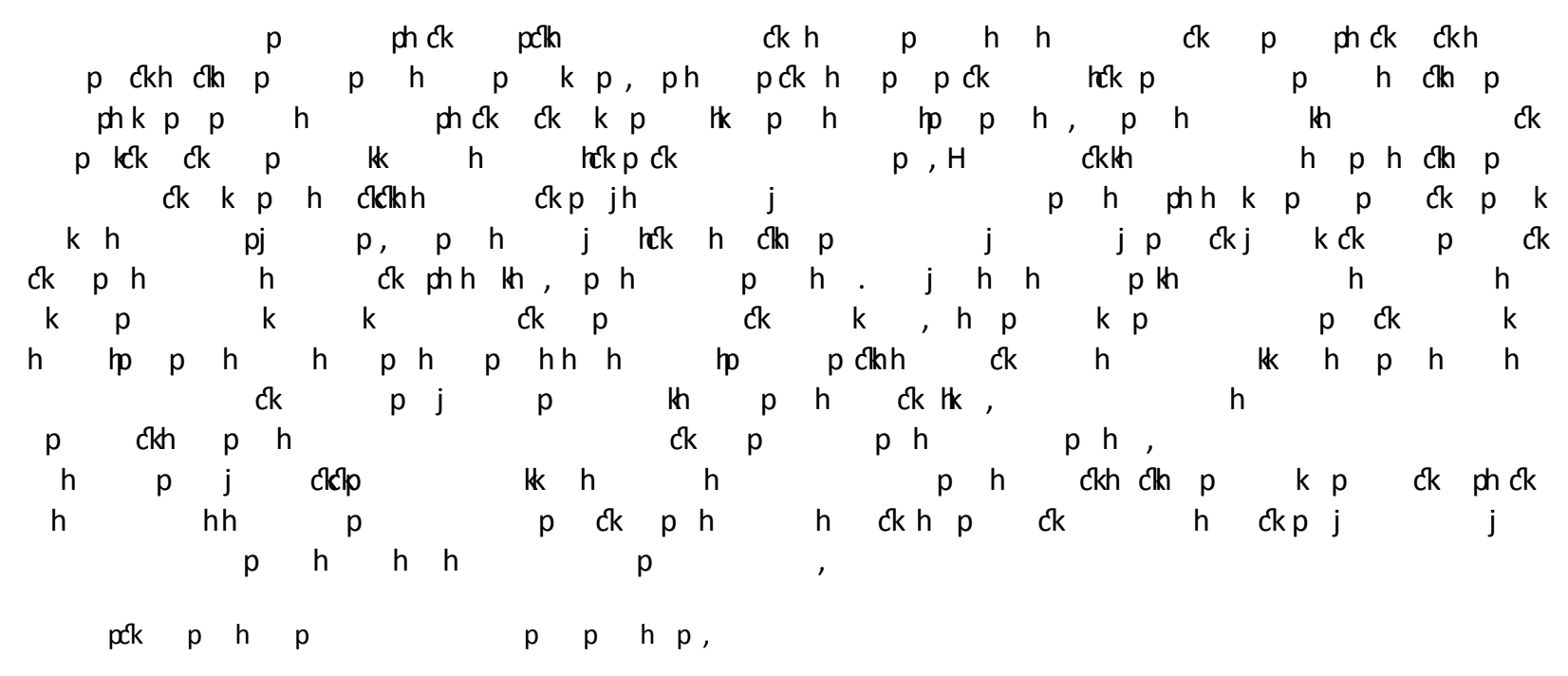

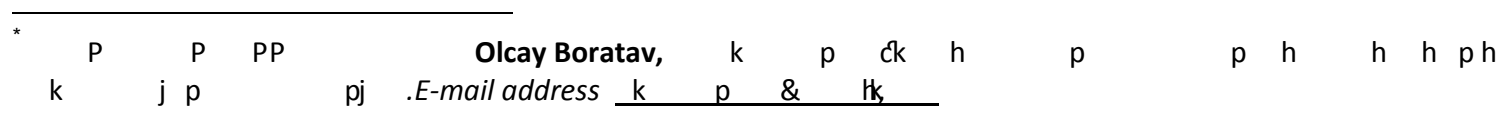




\section{Introduction}

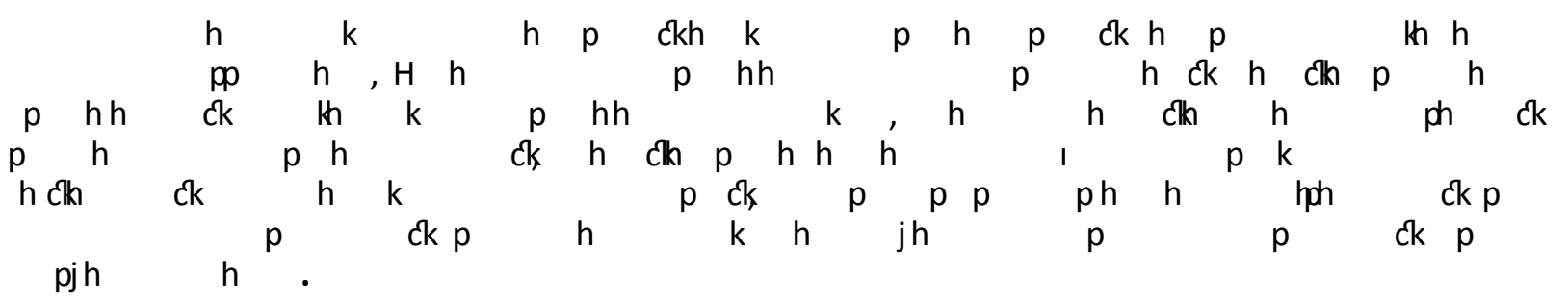

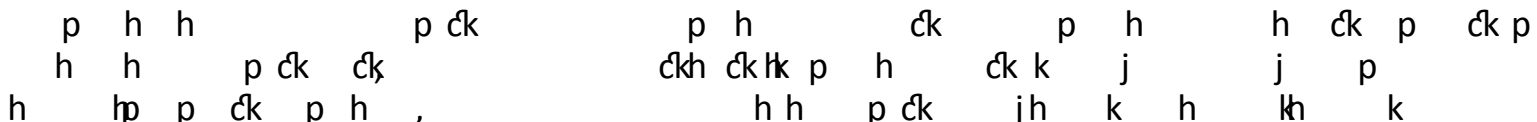

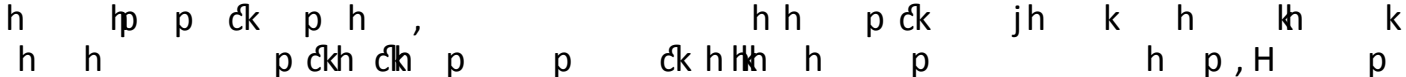

pp $p$ j ckh $p \quad k p$ ck ckth $\quad h \quad p$, tck $h \quad h \quad p$

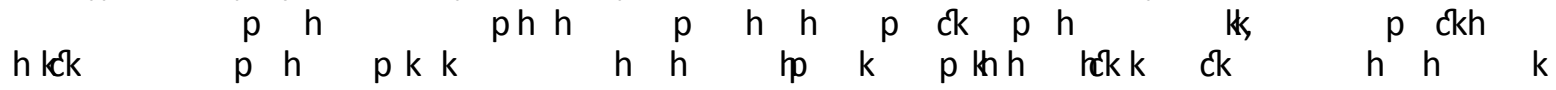
$h p h$ ph ck j,
$\mathrm{p} h$
$\mathrm{p} \quad \mathrm{ph} \mathrm{h} \quad \mathrm{jh}$
h $\mathrm{h} \quad \mathrm{k} \quad \mathrm{h}$
p ck h
ck

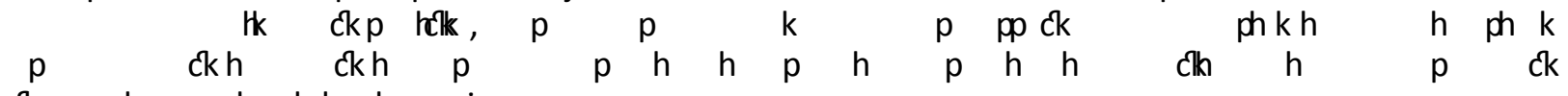
ck $p h \quad h \quad h h \quad h \quad j \quad p$,

\section{Ceramics at visual and intellectual expression}

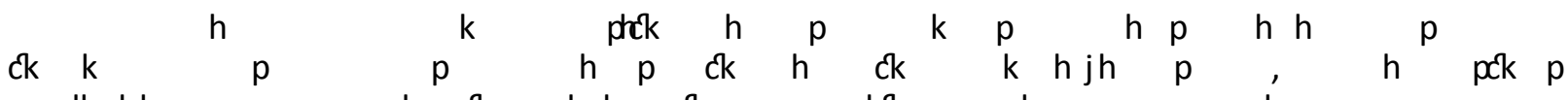

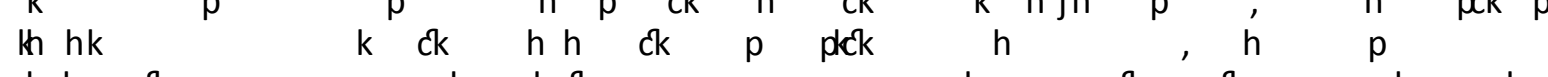

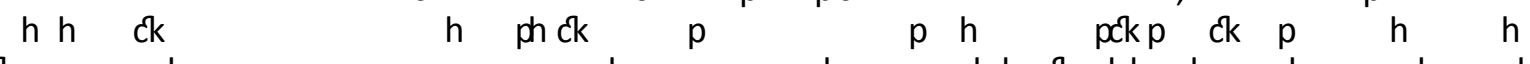
ck $h, \quad p \quad k \quad h, \quad h h c k h p k p h \quad h \quad h \quad h$ $p$ ck $p h \quad h \quad h \quad p k c k p k \quad h \quad h \quad h \quad h \quad p \quad p, P h \quad k$ $h$ ck k p ckh $h_{k}$ ckh $h k$ p p pkck

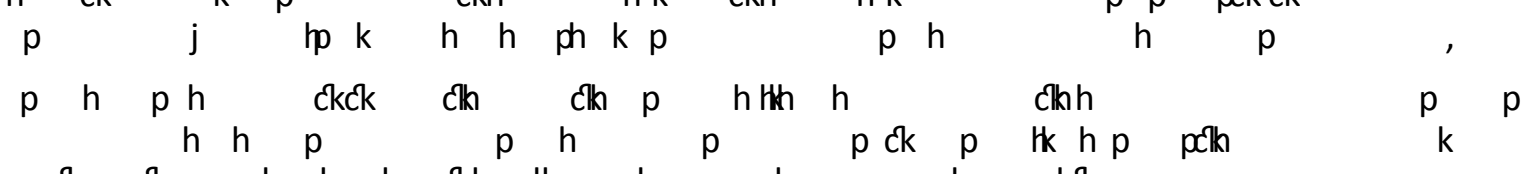

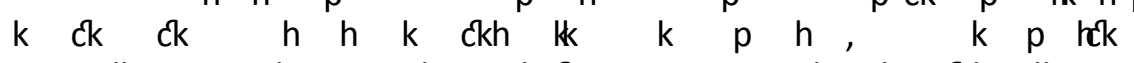

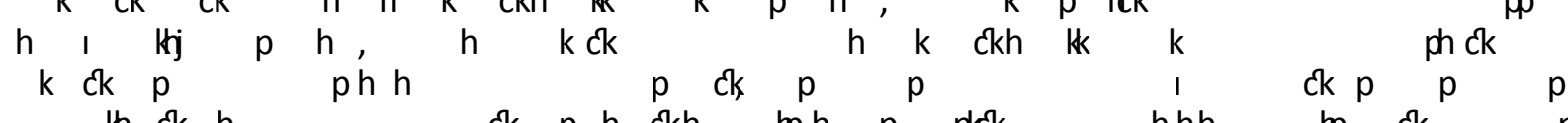

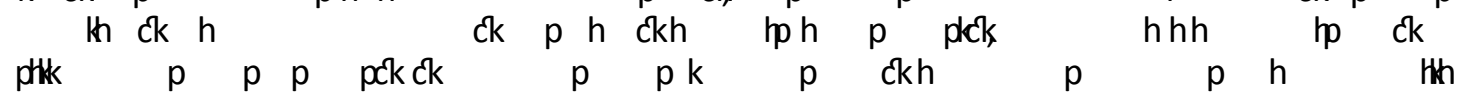
$p h \quad p h p p k$ p ckh $p h$,

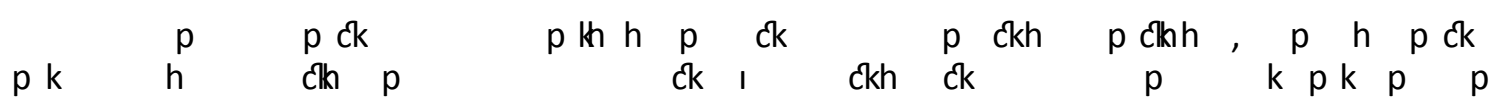
$h$ hth $h$ h $k$ h $h$ ph ck, H pckp $p$ lck $h \quad k \quad h \quad h \quad c k h$

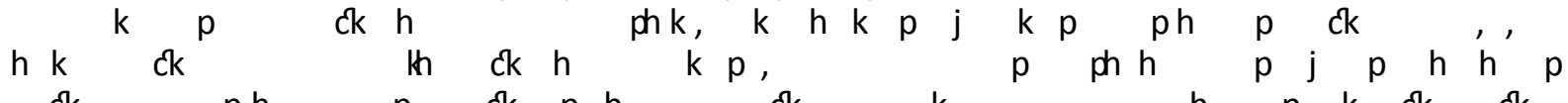

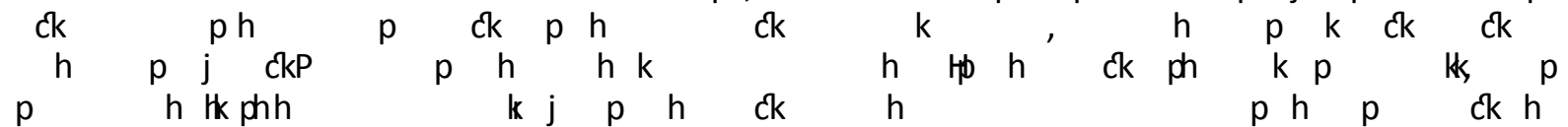




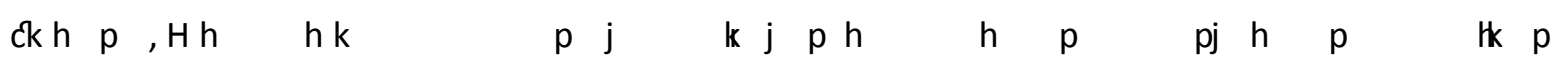

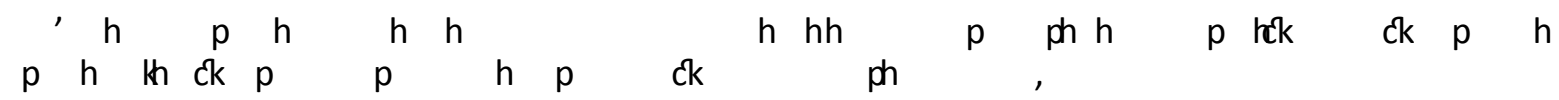
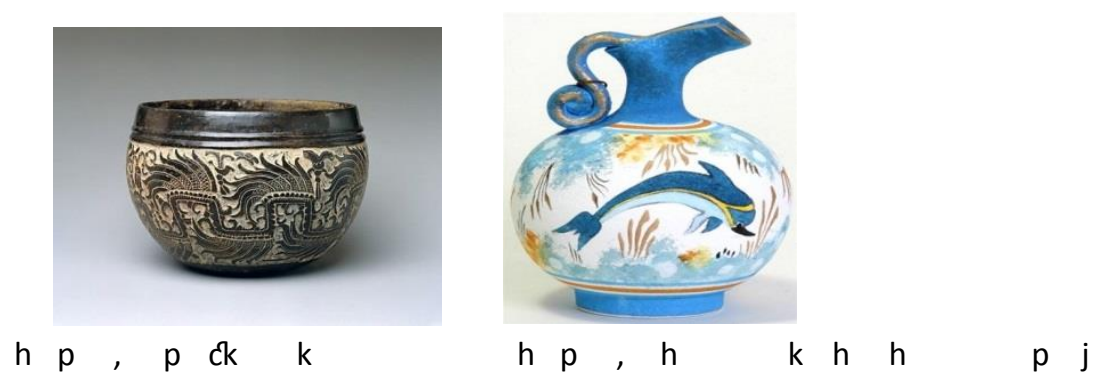

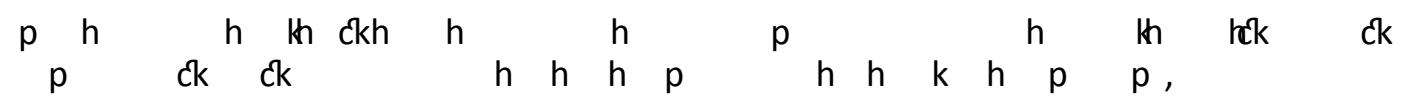

\section{Art conveyance and Creativity}

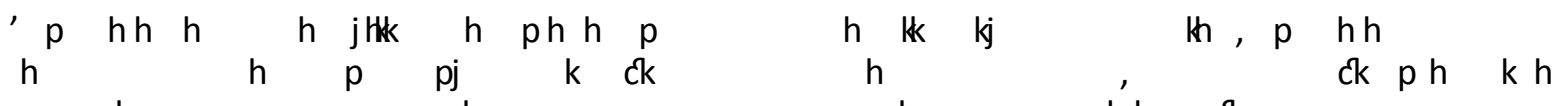
$p h p \quad p h \quad p p h \quad p h$ ck

$p \quad h h \quad p \quad$ ph $c k \quad p \quad p h h \quad h c k$ pj pph ck, pj pph $c k$

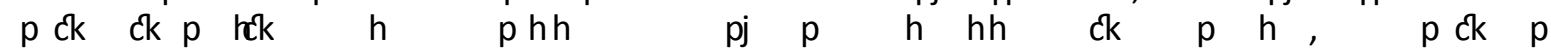
$p \quad h \quad k h \quad p j, h H h$ ck $k$ ckh $p \quad c k p h h$ jh ,

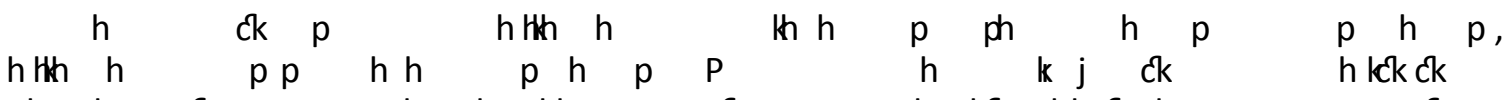
$k$ k p ck $p h$ h kh ck l, h tek hhck h p p ck j $h \mathrm{p} h \mathrm{~h} \quad \mathrm{p} \quad$.

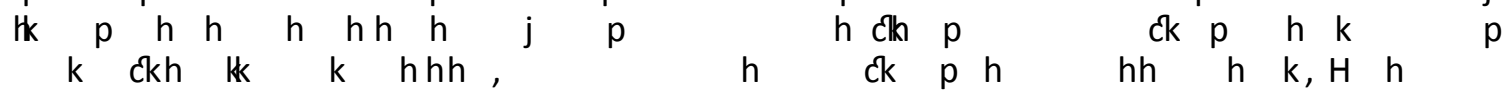

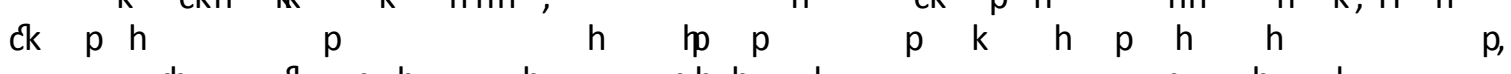
ph $c k p h \quad h \quad p h h k$ p $\quad h \quad k \quad h$ $h \mathrm{~h} k \mathrm{ckh} h$ ph ckh pck ck

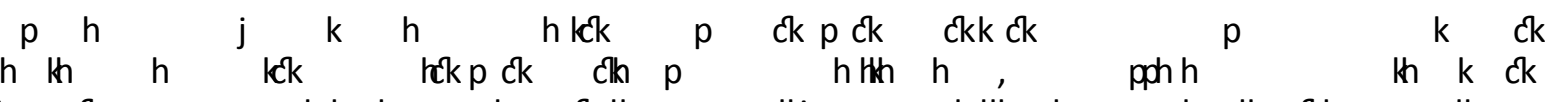

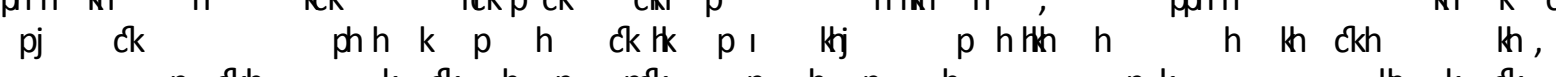

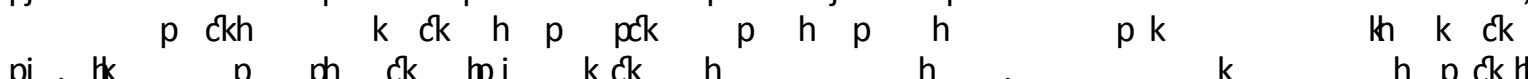

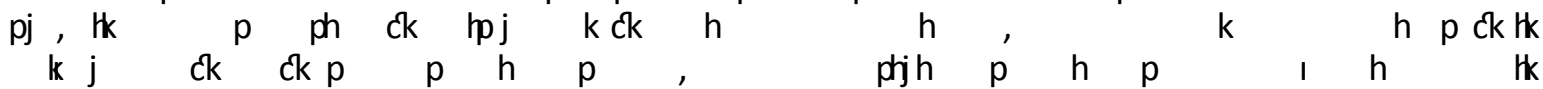
h ck h h $k$ ck k h h h HClk kj pjh hh h ph th $h$. $h$ hh ph ph $p h$ ck tk ph p ck h $h$

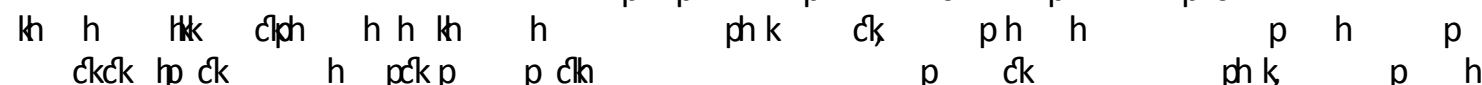

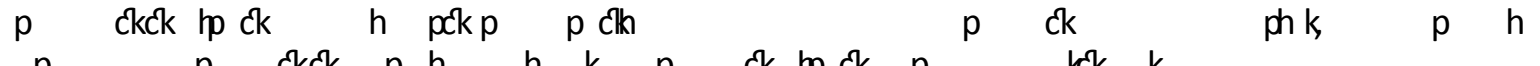

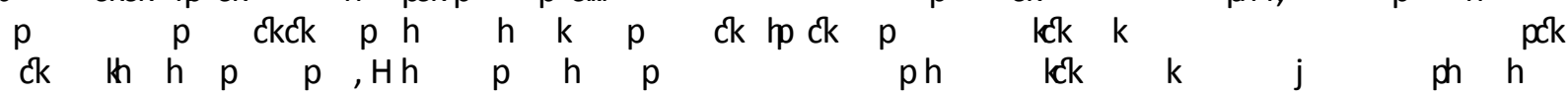




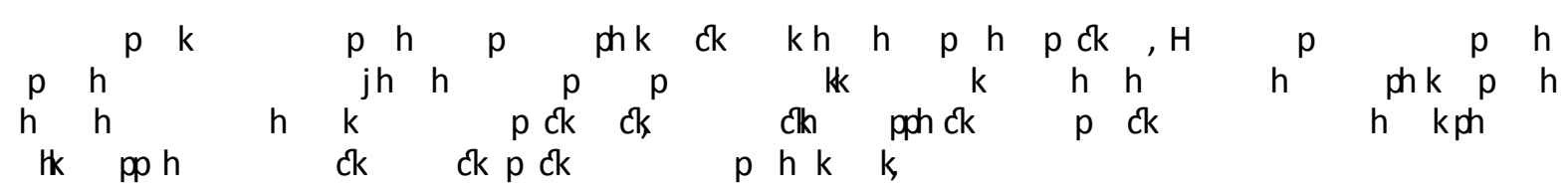

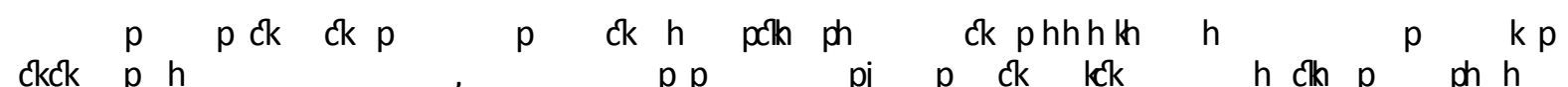

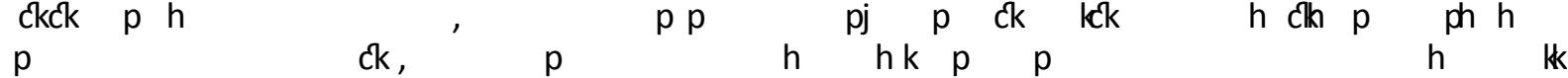
p phkpk $\quad p c k \quad h \quad h \quad p \quad c k$ ckh hkk $p h$.

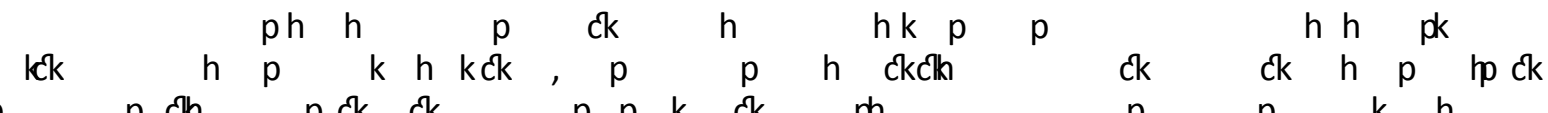

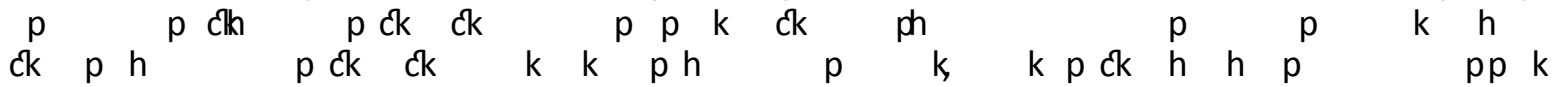

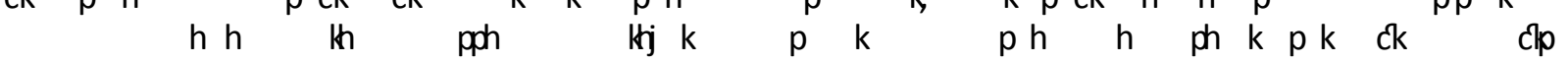
$p c k h, k \quad p \quad c k h \quad c k c k p h \quad h \quad c k$ ck $p$ h p ck $h$ $p$ ck $h$ Clk $c k \quad p \quad p \quad h \quad h \quad p \quad h \quad h \quad$ clkp $p h \quad p \quad h$ $p h \quad p p \quad p h \quad h \quad h \quad k p h \quad c k \quad p p \quad p \quad h \quad h \quad p h$

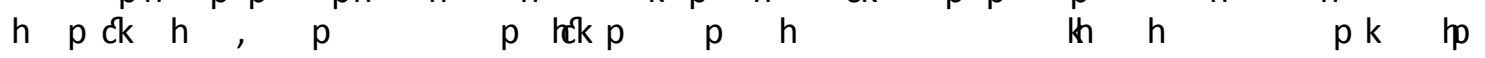
$\mathrm{p} \quad \mathrm{p} \quad \mathrm{ph} \quad \mathrm{p} k \quad \mathrm{~h} h$ phih ck $\mathrm{p} \quad \mathrm{h} k \quad \mathrm{H} \quad \mathrm{ck}$

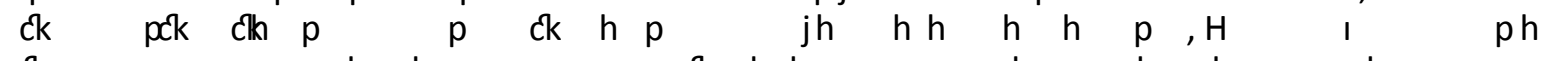

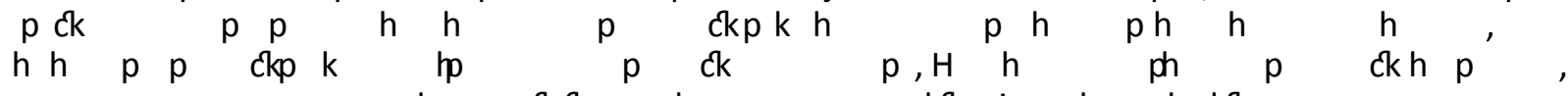

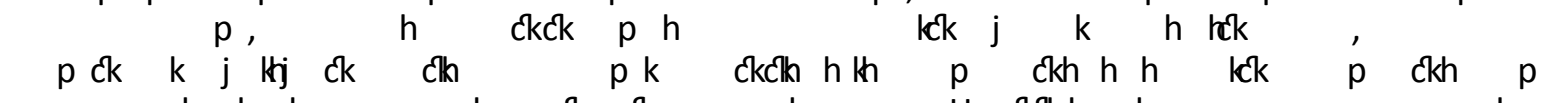

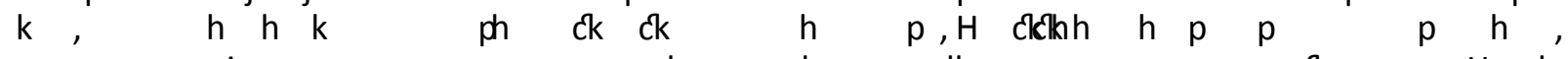
$\begin{array}{llllllllllll}p & p & p & p & h & p k & p & h & h & h\end{array}$

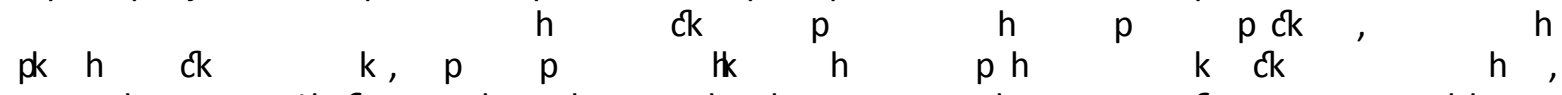

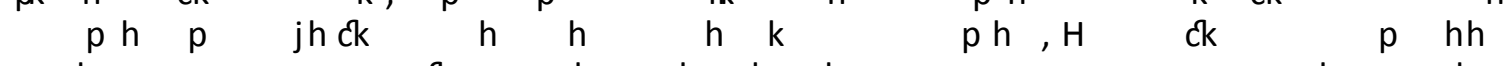
$\begin{array}{lllllllllllll}p & h & p & p & c k & p & h & h & h & p & p h\end{array}$

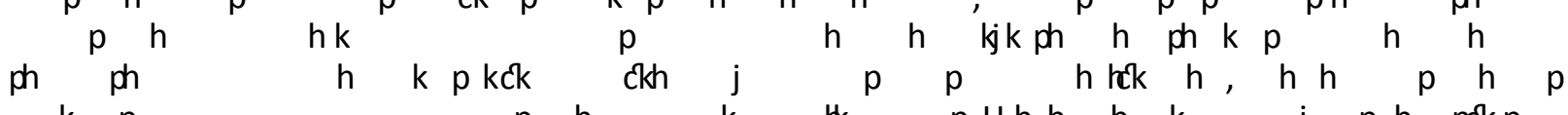

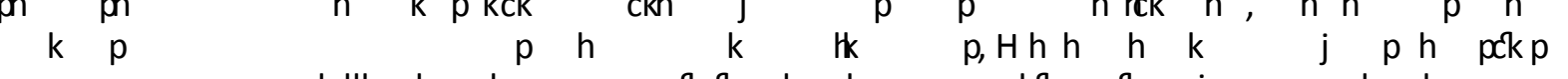

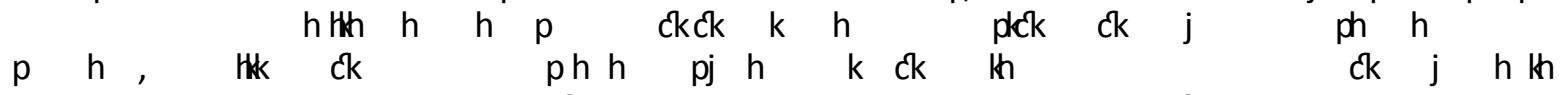

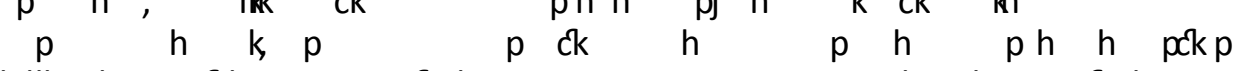

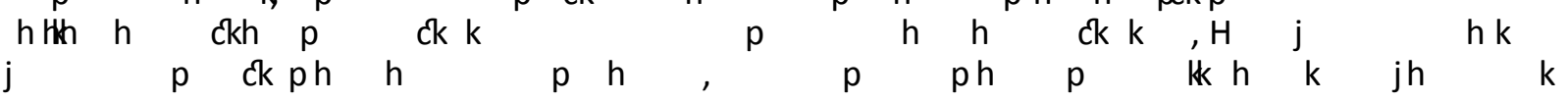
$h j \quad p \quad$ pj $p$,

\section{Art conveyance and ceramics}

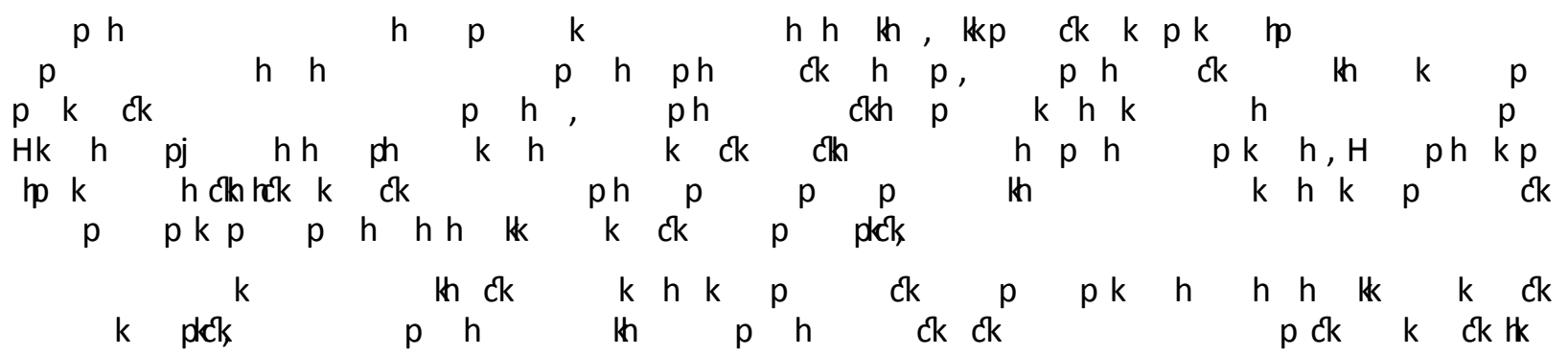




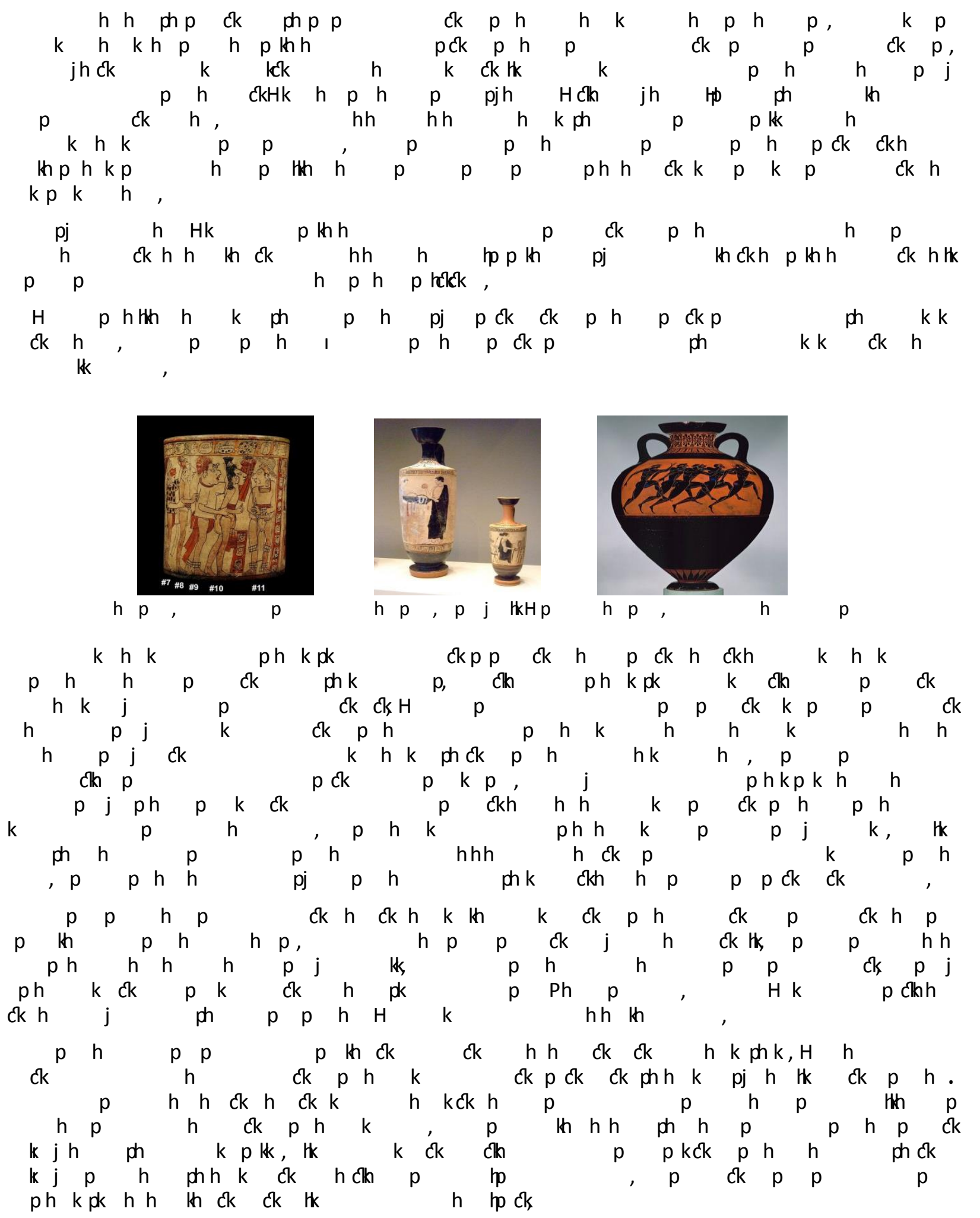



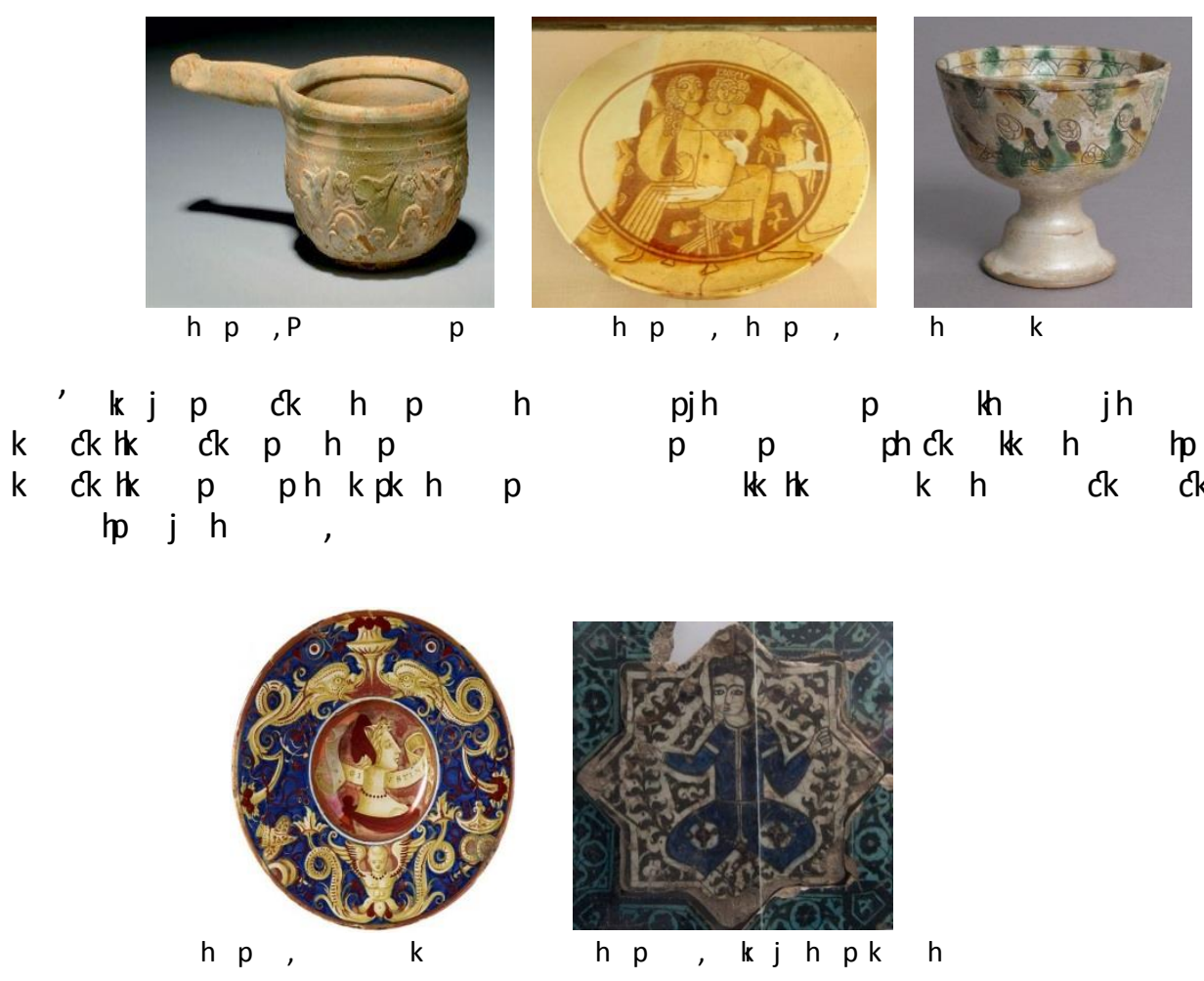

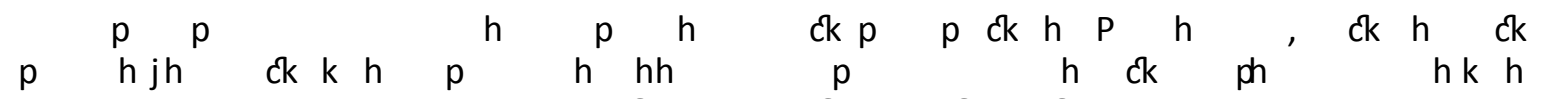

$$
\begin{aligned}
& \mathrm{h} h \mathrm{~h} p \mathrm{p}, \mathrm{ph} \quad \mathrm{ck} p \text { ck } \mathrm{pk} \text { ck h } \mathrm{p} h, \mathrm{~h} \\
& p \text { ckh tek } h \text { h } p \quad p \text { ckh } h \text {, } p \\
& \mathrm{~h} h \mathrm{~h} p \mathrm{ph}, \quad \mathrm{h} \mathrm{h} \text { ck } \mathrm{p} k \text { ck h } \mathrm{h} \text { ckh } \mathrm{h}
\end{aligned}
$$

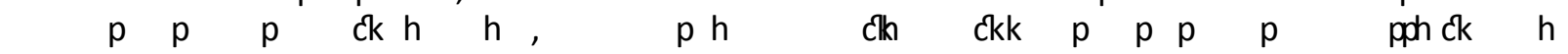

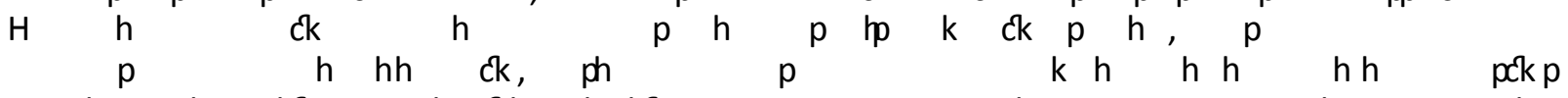

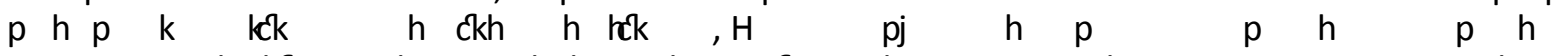

$$
\begin{aligned}
& \begin{array}{llllllllllll}
h \text { lck } & k & p k h & h & p c k H & h & k & p & p h
\end{array} \\
& \text { p h } h \quad h \quad h \quad h \quad c k \quad p
\end{aligned}
$$




\section{Conclusion}

$$
\begin{aligned}
& \text { h ph ckh } h \quad p \quad p \quad p h, h \quad p \quad h \quad \text { p } p h \\
& h h \quad p h \quad p h \quad h \quad h \quad h \quad h \quad h \quad p h \text { ckp } h \text { ph } \\
& \begin{array}{lllllllllllll}
p & p & p & p h & p & h & d k & h & c k & h & h & h
\end{array} \\
& k \quad p h \text { ckh } p \text { th } h, H^{2} \quad p h p k \text { jh thh ph } \\
& h \quad h h \quad p k \text { ck } h k \quad, H \quad h \text { ph } c k \text { p ck } p \quad p \quad \text { th } h
\end{aligned}
$$

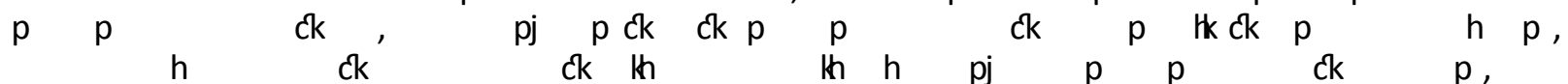

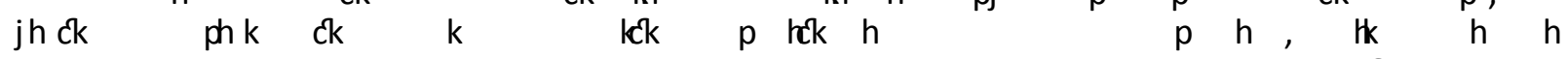

$$
\begin{aligned}
& \text { hhh } h \text { k } \quad p h \quad p \quad h \quad p \quad k \quad k h, \quad k \quad p \quad c k p h \\
& h \mathrm{p} p \mathrm{ph}, \mathrm{h} \quad \mathrm{k} \quad \mathrm{p} \text { k k } \mathrm{h} \text { k h , }
\end{aligned}
$$

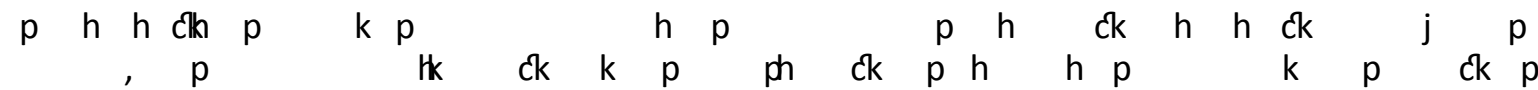

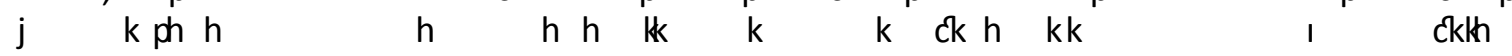

$$
\begin{aligned}
& p \quad p \quad p p h h \text { h p p ck ph } h \quad h p \text { ck k }
\end{aligned}
$$

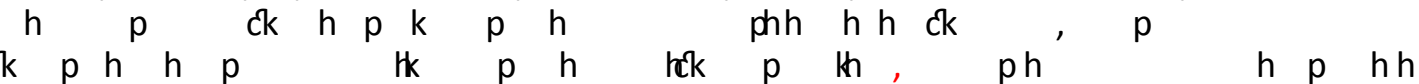

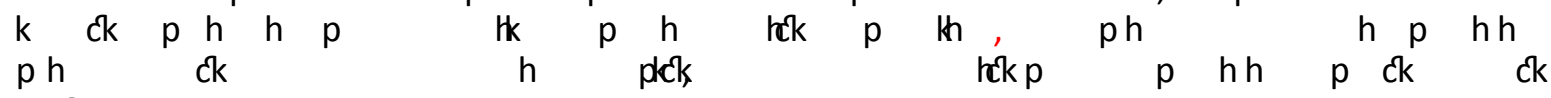

$$
\begin{aligned}
& p \text { pClk ph } p h \quad p \quad p \quad p h \quad p h h \quad p \quad \text { h p p jh } \\
& \text { ph } h \text { h } h \text { k } k \text { k } h \text { h kck, }
\end{aligned}
$$

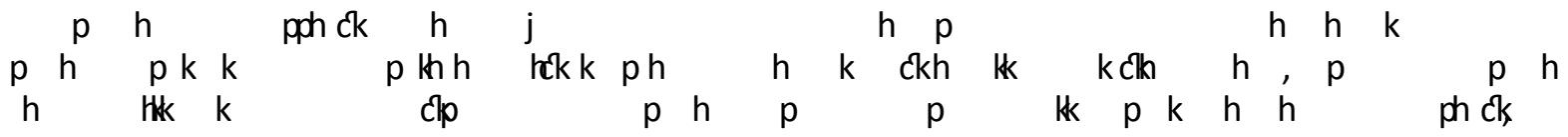

\section{References}

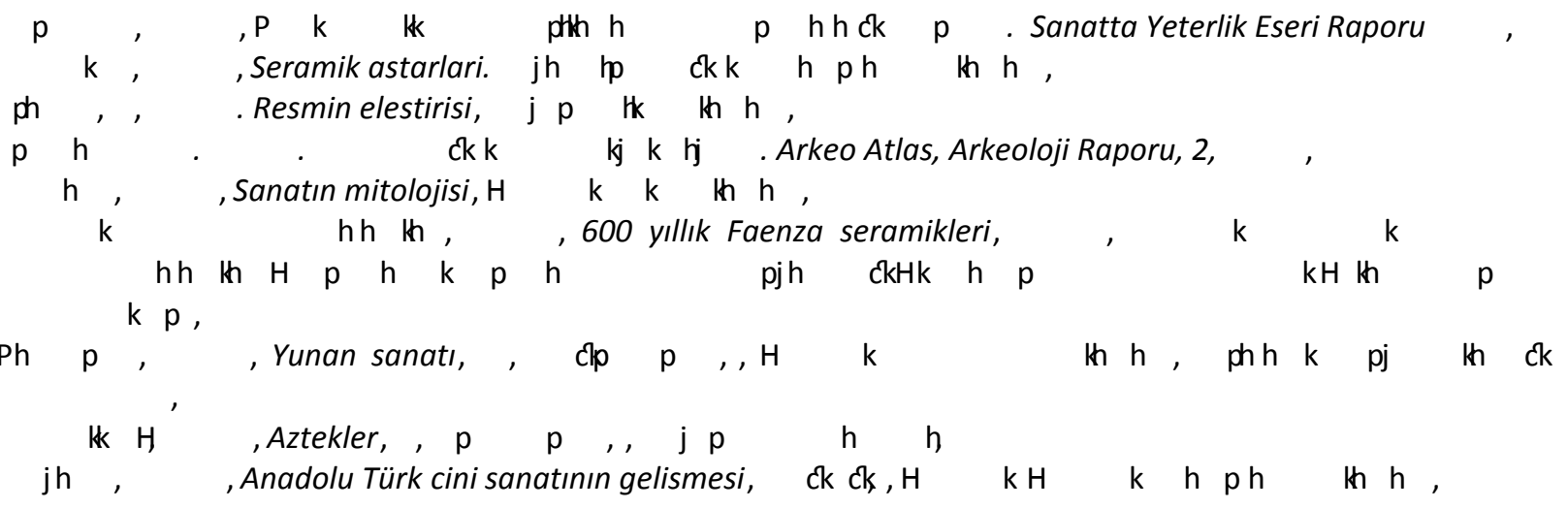

